\title{
THE ISOTOPY PROBLEM FOR JORDAN MATRIX ALGEBRAS
}

BY

HOLGER P. PETERSSON

\begin{abstract}
Conditions are given which are necessary and sufficient for two members of a certain class of Jordan matrix algebras to be isotopic. The main ingredient of these conditions is McCrimmon's notion of isotopy for alternative algebras.
\end{abstract}

Introduction. By a classical result due to Jacobson [2, IX, Theorem 2] (see also Faulkner [1, Theorem 1.8]), two reduced exceptional simple Jordan algebras are isotopic if and only if their corresponding coordinate algebras are isomorphic. It is the purpose of the present paper to extend this theorem to a larger class of Jordan matrix algebras. Using ideas of McCrimmon [6], it is fairly easy to give sufficient conditions for two such algebras to be isotopic (corollary of Proposition 3). That, under suitable restrictions, these conditions are also necessary will then follow from a conjugacy theorem for orthogonal systems of local idempotents in Jordan pairs recently established in [7].

The author would like to thank M. Koecher, O. Kühn, K. McCrimmon, and $\mathrm{A}$. Thedy for useful discussions on the subject.

0. Notations. Throughout $k$ is a unital commutative associative ring of scalars. All (nonassociative) algebras $C$ over $k$ are supposed to contain an identity, which we shall write as 1 . The nucleus of $C$ will be denoted by $\mathrm{N}(C)$; it is an associative subalgebra of $C$ containing 1 . If invertibility makes sense in $C$, the set of invertible elements will be denoted by $C^{\times}$. Given an involution $J$ of $C$, we put $\bar{x}=J(x)$ for $x \in C$ as long as no confusion can arise, and denote by $\mathrm{H}(C, J)$ the set of elements in $C$ fixed under $J$. For $a \in \mathrm{N}(C)^{\times} \cap \mathrm{H}(C, J)$, the map $J^{[a]}: C \rightarrow C$ defined by $J^{[a]}(x)=a \bar{x} a^{-1}$ for $x \in C$ is clearly an involution satisfying $\mathrm{H}\left(C, J^{[a]}\right)=\mathrm{H}(C, J) a^{-1}$. A submodule $C_{0}$ of $\mathrm{N}(C)$ is said to be $J$-ample in case $a C_{0} \bar{a} \subset C_{0}$ for all $a \in C$.

1. Coordinate triples and Jordan matrix algebras. Let $\left(C, J, C_{0}\right)$ be a coordinate triple, consisting, by definition, of an alternative algebra $C$ over $k$, an involution $J$ of $C$, and a $J$-ample submodule $C_{0}$ of $C$ satisfying $1 \in C_{0} \subset$ $\mathrm{N}(C) \cap \mathrm{H}(C, J)$. As is well known and easily seen, this implies $C_{0}=\mathrm{H}(C, J)$

Received by the editors November 23, 1976

AMS (MOS) subject classifications (1970). Primary 17C50; Secondary 17D05.

Key words and phrases. Jordan matrix algebras, isotopy, alternative algebras, coordinate triples, complete Jordan pairs. 
$\subset \mathrm{N}(C)$ if 2 happens to be invertible in $k$, and, for arbitrary base rings, the "maximal choice" for $C_{0}$, that is, $C_{0}=\mathrm{N}(C) \cap \mathrm{H}(C, J)$, satisfies the above requirements [3, p. 1.47]. Let $m>3$ be an integer and $g=\operatorname{diag}\left(g_{1}, \ldots, g_{m}\right)$ be an admissible diagonal matrix for $\left(C, J, C_{0}\right)$, that is, $g_{i} \in C_{0} \cap C^{\times}$for $1<i<m$. Then $A=\mathrm{H}_{m}\left(C, J, C_{0}, g\right)$ stands for the $k$-module of all $m$-by- $m$ matrices $X=\left(x_{i j}\right)$ with entries in $C$ satisfying $X=g^{t} \overline{X g}^{-1}$, where ${ }^{t} \bar{X}$ is the conjugate transpose of $X$, and $x_{i i} \in C_{0} g_{i}^{-1}$ for $1<i<m$. In the sequel it is to be understood that we consider the $k$-module $A$ only when $m=3$ or $C$ is associative. Following [3], [5], $A$ then carries canonically the structure of a unital quadratic Jordan algebra and is thus called a Jordan matrix algebra. More precisely, writing $e_{i j}$ for the ordinary matrix units and setting

$$
\alpha[i i]=\alpha e_{i i}, \quad a[i j]_{g}=g_{i} a e_{i j}+g_{j} \bar{a} e_{j i} \quad(i \neq j)
$$

for $\alpha, a \in C$, the elements $1[i i](1<i<m)$ make up a complete orthogonal system of idempotents in $A$ with corresponding Peirce spaces $A_{i i}=C_{0} g_{i}^{-1}[i i]$ and $A_{i j}=C[i j]_{g}(i \neq j)$. Also, if $P_{g}$ and \{\}$_{g}$ stand for the quadratic representation and triple product, respectively, of $A$, the following relations hold, for $\alpha, \beta \in C_{0}, a, b \in C$ and $i, j, l \in\{1, \ldots, m\}$ mutually distinct.

$$
\begin{gathered}
P_{g}\left(\alpha g_{i}^{-1}[i i]\right) \beta g_{i}^{-1}[i i]=\alpha\left(g_{i}^{-1} \beta g_{i}^{-1}\right) \alpha g_{i}^{-1}[i i], \\
P_{g}\left(a[i j]_{g}\right) \alpha g_{i}^{-1}[i i]=g_{j}(\bar{a} \alpha a)[j j], \\
P_{g}\left(a[i j]_{g}\right) b[i j]_{g}=a\left(g_{j} \bar{b} g_{i}\right) a[i j]_{g} \\
\left\{\alpha g_{i}^{-1}[i i] \beta g_{i}^{-1}[i i] a[i j]_{g}\right\}_{g}=g_{i}^{-1} \alpha g_{i}^{-1} \beta a[i j]_{g} \\
\left\{\alpha g_{i}^{-1}[i i] a[i j]_{g} \beta g_{j}^{-1}[i j]\right\}_{g}=\left(g_{i}^{-1} \alpha\right) a\left(\beta g_{j}^{-1}\right)[i j]_{g} \\
\left\{a[i j]_{g} \alpha g_{j}^{-1}[i j] b[j l]_{g}\right\}_{g}=a \alpha b[i l]_{g}
\end{gathered}
$$

If $g=e$, the $m$-by- $m$ identity matrix, the subscript " $g$ " will be dropped from the above notation, and we shall write $\mathrm{H}_{m}\left(C, J, C_{0}\right)$ instead of $\mathrm{H}_{m}\left(C, J, C_{0}, e\right)$. Our aim here is to describe the isotopy class of $A$ in terms of the parameters $C, J, C_{0}, g$. (It does not seem to make much sense to include $m$ as an additional parameter since, for example, the assumption that $C$ be strictly alternative forces $m=3$ anyway.) According to [5], the rule $X \mapsto X g$ defines an isomorphism from $A$ onto $\mathrm{H}_{m}\left(C, J, C_{0}\right)^{(y)}$ where $y=g^{-1}$. Hence, in dealing with the isotopy problem, we are always allowed to assume $g=e$.

2. Isotopes of coordinate triples. Morphisms of coordinate triples are defined in the obvious way. Given a coordinate triple $\left(C, J, C_{0}\right)$ and $a \in C_{0}$ $\cap C^{\times}$, it follows easily that $\left(C, J, C_{0}\right)^{[a]}=\left(C, J^{[a]}, C_{0}^{[a]}\right)$, where $C_{0}^{[a]}=$ $C_{0} a^{-1}$, is again a coordinate triple. We now proceed to define isotopes of coordinate triples. Recall that, if $u, v$ are invertible elements of an alternative 
algebra $C$ over $k$, the $u, v$-isotope, $C^{(u, v)}$, of $C$ in the sense of McCrimmon [6] is the $k$-module $C$ together with a new product defined by $x{ }_{u, v} y=(x u)(v y)$ for $x, y \in C . C^{(u, v)}$ is again alternative, with identity element $1^{(u, v)}=(u v)^{-1}$.

Now suppose $\left(C, J, C_{0}\right)$ is a coordinate triple. Fixing $u, v \in C^{\times}$, we define $J^{(u, v)}: C^{(u, v)} \rightarrow C^{(u, v)}$ by

$$
J^{(u, v)}(x)=\bar{x}^{(u, v)}=\overline{x(u v)}(u v)^{-1}
$$

for $x \in C$ and set $C_{0}^{(u, v)}=C_{0}(u v)^{-1}$. We wish to show, among other things, that $\left(C^{(u, v)}, J^{(u, v)}, C_{0}^{(u, v)}\right)$ is a coordinate triple. To this end we require the Moufang Identities

$$
\begin{aligned}
& (a b a) x=a(b(a x)), \\
& x(a b a)=((x a) b) a, \\
& a(x y) a=(a x)(y a),
\end{aligned}
$$

valid in every alternative algebra. As all norms $n(x)=x \bar{x}$, hence all traces $x+\bar{x}$, are in the nucleus, we have, following [3, p. 1.47],

$$
n(x y)=x n(y) \bar{x} .
$$

This immediately implies

$$
x^{-1} n(x y)=n(y) \bar{x},
$$

hence, in particular,

$$
x^{-1} n(x)=\bar{x}
$$

for $x \in C^{\times}$. We shall also have occasion to consider the unital quadratic Jordan algebra $C^{+}$attached to $C$, whose quadratic representation will be denoted by $P$. Thus $P(x)=L_{x} R_{x}, L, R$ being the left, right multiplication, respectively, of $C$, and

$$
P(x y)=R_{y} P(x) L_{y},
$$

by [6, (8)]. If any of the above notation is supposed to refer to $C^{(u, v)}$ rather than $C$, this will be indicated by the superscript " $(u, v)$ ". For example, given $x \in C^{\times}, x^{-1(u, v)}$ stands for the inverse of $x$ in $C^{(u, v)}$. It is related by

$$
x^{-1(u, v)}=P\left((u v)^{-1}\right) x^{-1}
$$

to the inverse of $x$ in $C$. We now claim

$$
\bar{x}^{(u, v)}=P\left((u v)^{-1}\right)(n(u v) \bar{x})=\left[\overline{(x u) v} v^{-1}\right] u^{-1} \text {. }
$$

Indeed,

$$
\begin{aligned}
\bar{x}^{(u, v)} & =(\overline{u v} \bar{x})(u v)^{-1} \\
& =(u v)^{-1}(n(u v) \bar{x})(u v)^{-1} \quad(\text { by }(12)),
\end{aligned}
$$

giving the first part of (15). As to the second, we apply (13) and obtain 


$$
\begin{aligned}
\bar{x}^{(u, v)} & =P\left(v^{-1} u^{-1}\right)(n(u v) \bar{x}) \\
& =\left(v^{-1}\left[u^{-1} n(u v) \bar{x}\right] v^{-1}\right) u^{-1} \\
& =\left(v^{-1}[n(v) \bar{u} \bar{x}] v^{-1}\right) u^{-1} \quad(\text { by }(10)) \\
& =\left([\bar{v}(\bar{u} \bar{x})] v^{-1}\right) u^{-1} \quad(\text { by }(12)),
\end{aligned}
$$

which completes the proof of (15). Next we show

$$
R_{0} J^{(\text {ouv }, 1)}=J^{(u, v)} R_{b}, \quad R_{0} C_{0}^{(\text {vuro, }, 1)}=C_{0}^{(u, v)} .
$$

Abbreviating $z=v u v$ gives

$$
\begin{aligned}
& \bar{x}^{(z, 1)} v=\left(\overline{x z} z^{-1}\right) v \\
& =\left(\left[\left(\overline{x z} v^{-1}\right) u^{-1}\right] v^{-1}\right) v \quad(\text { by }(8)) \\
& =\left[\overline{((x v) u) v} v^{-1}\right] u^{-1} \quad(\text { by }(8)) \\
& =\overline{x v}^{(u, v)} \quad(\text { by }(15))
\end{aligned}
$$

and

$$
C_{0}^{(z, 1)} v=C_{0} z^{-1} v=C_{0}\left(v^{-1} u^{-1}\right),
$$

as desired. Finally, let us mention

$$
n^{(u, 1)}(x)=n(x u) u^{-1},
$$

which follows from

$$
\begin{aligned}
n^{(u, 1)}(x) & =x \cdot{ }_{(u, 1)} \bar{x}^{(u, 1)}=(x u)\left(\overline{x u} u^{-1}\right) \\
& =[(x u) \overline{x u}] u^{-1} .
\end{aligned}
$$

Proposition 1. (i) Let $C$ be an alternative algebra over $k$ and $u, v \in C^{\times}$. Then $\mathrm{N}\left(C^{(u, v)}\right)=\mathrm{N}(C)(u v)^{-1}$.

(ii) Let $\left(C, J, C_{0}\right)$ be a coordinate triple and $u, v \in C^{\times}$. Then

$$
\left(C, J, C_{0}\right)^{(u, v)}=\left(C^{(u, v)}, J^{(u, v)}, C_{0}^{(u, v)}\right)
$$

is a coordinate triple, called the $u$, v-isotope of $\left(C, J, C_{0}\right)$.

Proof. (i) Put $z=v u v$. By [6, Proposition 6], $R_{v}$ is an isomorphism from $C^{(z, 1)}$ onto $C^{(u, v)}$. This implies $\mathrm{N}\left(C^{(u, v)}\right)=\mathrm{N}\left(C^{(2,1)}\right) v$, whence it suffices to consider the case $v=1$. Then observe that, in any alternative algebra, the three defining associator relations for the nucleus collapse to a single one. For $a, b, c \in C$, denote by $[a, b, c]$ their associator in $C$ and by $[a, b, c]^{(u, 1)}$ their associator in $C^{(u, 1)}$. Subtracting and adding the term $[(a u)(b u)] c$ from and to $[a, b, c]^{(u, 1)}$ yields

$$
[a, b, c]^{(u, 1)}=[a u, b, u] c+[a u, b u, c]
$$


Hence $a u \in \mathrm{N}(C)$ implies $a \in \mathrm{N}\left(C^{(u, 1)}\right)$. Conversely, if $a \in \mathrm{N}\left(C^{(u, 1)}\right)$, we set $c=1$ in (18), obtain $[a u, b, u]=0(b \in C)$ and then $[a u, b u, c]=0$ for all $b$, $c \in C$. This yields $a u \in \mathrm{N}(C)$ and completes the proof of (i).

(ii) By (16) and [6, Proposition 6], we may assume $v=1$. Then $\bar{x}^{(u, 1)} u=\overline{x u}$, hence

$$
\bar{x}^{-(u, 1)}=\overline{\bar{x}^{(u, 1)} u} u^{-1}=(x u) u^{-1}=x,
$$

so $J^{(u, 1)}$ has period at most 2. For $x, y \in C$, we obtain

$$
\begin{aligned}
\bar{y}^{(u, 1)}{ }_{u, 1} \bar{x}^{(u, 1)} & =\left\{\left[P\left(u^{-1}\right)(n(u) \bar{y})\right] u\right\} \bar{x}^{(u, 1)} \\
& =\left(u^{-1} n(u) \bar{y}\right) \bar{x}^{(u, 1)} \\
& =(\bar{u} \bar{y})(\overline{x u} \bar{u}) n(u)^{-1} \quad(\text { by }(12)) \\
& =[\bar{u}(\bar{y} \overline{x u}) \bar{u}] n(u)^{-1} \quad(\text { by }(9)) \\
& =\left(\bar{u}(\overline{(x u) y}) u^{-1}={\overline{x \cdot{ }_{u, 1} y}}^{(u, 1)} .\right.
\end{aligned}
$$

Hence $J^{(u, 1)}$ is an involution of $C^{(u, 1)}$. Clearly, an element $x \in C$ remains fixed under $J^{(u, 1)}$ if and only if $x u=\overline{x u}$. This implies $\mathrm{H}\left(C^{(u, 1)}, J^{(u, 1)}\right)=$ $\mathrm{H}(C, J) u^{-1}$, and we may conclude from (i) that $C_{0}^{(u, 1)}=C_{0} u^{-1}$ is a submodule of $\mathrm{N}\left(C^{(u, 1)}\right) \cap \mathrm{H}\left(C^{(u, 1)}, J^{(u, 1)}\right)$ containing the identity of $C^{(u, 1)}$. It remains to prove that $C_{0}^{(u, 1)}$ is $J^{(u, 1)}$-ample. Observing the identity

$$
(a b \bar{a}) x=a(b(\bar{a} x))
$$

for $a, x \in C, b \in C_{0}$, which follows easily from (7), we obtain

$$
\begin{aligned}
x \cdot_{u, 1} C_{0}^{(u, 1)}{ }_{u, 1} \bar{x}^{(u, 1)}=\left\{\left[(x u) C_{0} u^{-1}\right] u\right\} \bar{x}^{(u, 1)} \\
=(x u)\left(C_{0}\left(\overline{x u} u^{-1}\right)\right)=\left[(x u) C_{0} \overline{x u}\right] u^{-1} \\
\subset C_{0} u^{-1}
\end{aligned}
$$

The proof of Proposition 1 is now complete.

It seems remarkable that, if $(C, J)$ is an alternative algebra with involution not necessarily arising from a coordinate triple, and if $u, v$ are invertible elements of $C$, the mapping $J^{(u, v)}$ defined above need not be an involution of $C^{(u, v)}$. To see this, consider McCrimmon's [6] example of the free commutative alternative algebra $C$ in three generators $x, y, z$ over the integers subject to the relation $z^{3}=0$, and put $u=1+z$. Then $J=\mathrm{id}_{C}$ is an involution of $C$, whereas, since $C^{(u, 1)}$ is no longer commutative, $J^{(u, 1)}=\mathrm{id}_{C^{(u, 1)}}$ cannot be an involution of $C^{(u, 1)}$.

Propostrion 2. Let $\left(C, J, C_{0}\right)$ be a coordinate triple and $u, v \in C^{\times}$.

(i) If also $u^{\prime}, v^{\prime} \in C^{\times}$, we have 


$$
\left(C, J, C_{0}\right)^{(u, v)\left(u^{\prime}, v\right)}=\left(C, J, C_{0}\right)^{\left(u^{\prime \prime}, v^{*}\right)},
$$

where $u^{\prime \prime}=u\left(v u^{\prime}\right) u, v^{\prime \prime}=v\left(v^{\prime} u\right) v$.

(ii) If $a \in C_{0} \cap C^{\times}$, we have

$$
\left(C, J, C_{0}\right)^{[a](u, v)}=\left(C, J, C_{0}\right)^{(u, v)\left[a(u v)^{-1}\right]} .
$$

Proof. (i) From [6, (17)] we know $C^{(u, v)\left(u^{\prime}, v\right)}=C^{\left(u^{\prime \prime}, 0\right)}$, so we only have to worry about $J$ and $C_{0}$. By (16) and [6, Proposition 6], $R_{0}^{-1}$ is an isomorphism from $\left(C, J, C_{0}\right)^{(u, v)}$ onto $\left(C, J, C_{0}\right)^{(z, 1)}$, where $z=v u v$, hence also an isomorphism from

$$
\left(C, J, C_{0}\right)^{(u, v)\left(u^{\prime}, v^{\prime}\right)} \text { onto }\left(C, J, C_{0}\right)^{(z, 1)\left(u^{\prime} v^{-1}, v^{\prime} v^{-1}\right)} \text {. }
$$

Assuming for the moment that the case " $v=1$ " has been taken care of, we conclude

$$
\left(C, J, C_{0}\right)^{(z, 1)\left(u^{\prime} 0^{-1}, 0^{\prime} v^{-1}\right)}=\left(C, J, C_{0}\right)^{\left(u^{*}, 0^{*}\right)}
$$

where

$$
v^{*}=\left(v^{\prime} v^{-1}\right) z=\left(v^{\prime} u\right) v \quad(\text { by }(8))
$$

and

$$
\begin{aligned}
u^{*} & =z\left[\left(u^{\prime} v^{-1}\right) z\right]=(v u v)\left[\left(u^{\prime} u\right) v\right] \quad(\text { by }(8)) \\
& =v\left(u\left[v\left(u^{\prime} u\right) v\right]\right) \quad(\text { by }(7)) \\
& =v\left(u\left[\left(v u^{\prime}\right)(u v)\right]\right) \quad(\text { by }(9)) \\
& =v\left(\left[u\left(v u^{\prime}\right) u\right] v\right) \quad(\text { by }(7)) \\
& =v u^{\prime \prime} v
\end{aligned}
$$

This implies

$$
u^{*} v^{*}=\left(v u^{\prime \prime} v\right)\left[\left(v^{\prime} u\right) v\right]=v\left(u^{\prime \prime}\left[v\left(v^{\prime} u\right) v\right]\right) \quad(\text { by }(7)),
$$

hence

$$
u^{*} v^{*}=v\left(u^{\prime \prime} v^{\prime \prime}\right)
$$

Given $x \in C$, we now compute

$$
\begin{aligned}
R_{v} \bar{x}^{\left(u^{*}, v^{*}\right)} & =R_{v} P\left(\left(u^{*} v^{*}\right)^{-1}\right)\left(n\left(u^{*} v^{*}\right) \bar{x}\right) \quad(\text { by }(15)) \\
& =R_{v} P\left(\left(u^{\prime \prime} v^{\prime \prime}\right)^{-1} v^{-1}\right)\left(\left[v n\left(u^{\prime \prime} v^{\prime \prime}\right) \bar{v}\right] \bar{x}\right) \quad(\text { by }(20),(10)) \\
& =R_{v} R_{v}^{-1} P\left(\left(u^{\prime \prime} v^{\prime \prime}\right)^{-1}\right) L_{v}^{-1}\left(v n\left(u^{\prime \prime} v^{\prime \prime}\right) \overline{x v}\right) \quad(\text { by }(13),(19)) \\
& =\overline{x v}^{\left(u^{*}, v^{\prime \prime}\right)} .
\end{aligned}
$$

Also, 


$$
R_{v} C_{0}^{\left(u^{*}, v^{*}\right)}=R_{0} C_{0}\left(u^{\prime \prime} v^{\prime \prime}\right)^{-1} v^{-1}=C_{0}^{\left(u^{\prime \prime}, v^{\prime \prime}\right)} \quad \text { (by (20)). }
$$

Summing up, $R_{0}$ turns out to be an isomorphism from $\left(C, J, C_{0}\right)^{\left(u^{*}, 0^{*}\right)}$ onto $\left(C, J, C_{0}\right)^{\left(u^{\prime \prime}, v^{*}\right)}$, and it follows that we may assume $v=1$. Then, setting $w=u^{\prime} \cdot{ }_{u, 1} v^{\prime}=\left(u^{\prime} u\right) v^{\prime}, w^{-1(u, 1)}$ is the identity of $C^{(u, 1)\left(u^{\prime}, 0\right)}=C^{\left(u^{\prime \prime}, v^{\prime}\right)}$ and hence

$$
w^{-1(u, 1)}=\left(u^{\prime \prime} v^{\prime \prime}\right)^{-1} \text {. }
$$

This implies $w=\left[\left(u^{\prime \prime} v^{\prime \prime}\right)^{-1}\right]^{-1(u, 1)}$ and, in conjunction with (14), yields

$$
w=P\left(u^{-1}\right)\left(u^{\prime \prime} v^{\prime \prime}\right) \text {. }
$$

Now let $x \in C$. Then

$$
\begin{aligned}
\bar{x}^{(u, 1)\left(u^{\prime}, v^{\prime}\right)} & =P^{(u, 1)}\left(w^{-1(u, 1)}\right)\left(n^{(u, 1)}(w)_{u, 1} \bar{x}^{(u, 1)}\right) \quad(\text { by }(15)) \\
& =P\left(\left(u^{\prime \prime} v^{\prime \prime}\right)^{-1}\right) P(u)\left[\left(n(w u) u^{-1} u\right) \bar{x}^{(u, 1)}\right] \quad(\text { by }(21),(17)) \\
& =P\left(\left(u^{\prime \prime} v^{\prime \prime}\right)^{-1}\right) P(u)\left[n\left(u^{-1}\left(u^{\prime \prime} v^{\prime \prime}\right)\right) \bar{x}^{(u, 1)}\right] \quad(\text { by }(22)) \\
& =P\left(\left(u^{\prime \prime} v^{\prime \prime}\right)^{-1}\right) P(u)\left[\left(u^{-1} n\left(u^{\prime \prime} v^{\prime \prime}\right) \bar{u}^{-1}\right)\left((\bar{u} \bar{x}) u^{-1}\right)\right] \quad(\text { by }(10)) \\
& =P\left(\left(u^{\prime \prime} v^{\prime \prime}\right)^{-1}\right) P(u)\left[u^{-1}\left(n\left(u^{\prime \prime} v^{\prime \prime}\right) \bar{u}^{-1}(\bar{u} \bar{x})\right) u^{-1}\right] \quad(\text { by (9)) } \\
& =P\left(\left(u^{\prime \prime} v^{\prime \prime}\right)^{-1}\right)\left(n\left(u^{\prime \prime} v^{\prime \prime}\right) \bar{x}\right)=\bar{x}^{\left(u^{\prime \prime}, v^{\prime \prime}\right)} \quad(\text { by }(15)) .
\end{aligned}
$$

Finally,

$$
\begin{aligned}
C_{0}^{(u, 1)\left(u^{\prime}, v^{\prime}\right)} & =C_{0} u^{-1} \cdot u_{u, 1} w^{-1(u, 1)} \\
& =\left(C_{0} u^{-1} u\right)\left(u^{\prime \prime} v^{\prime \prime}\right)^{-1} \quad(\text { by }(21)) \\
& =C_{0}^{\left(u^{\prime \prime}, v^{\prime \prime}\right)},
\end{aligned}
$$

and the proof of (i) is complete.

(ii) As in (i), we first reduce to the case $v=1$ : Setting $b=a(u v)^{-1}$ and using the previous notation, $R_{0}^{-1}$ is an isomorphism from $\left(C, J, C_{0}\right)^{(a)(u, v)}$ onto $\left(C, J, C_{0}\right)^{[a(z, 1)}=\left(C, J, C_{0}\right)^{(z, 1)\left(b^{\prime}\right]}$, where $b^{\prime}=a z^{-1}$. On the other hand, $R_{0}$ is an isomorphism from $\left(C, J, C_{0}\right)^{(2,1)(b]}$ onto $\left(C, J, C_{0}\right)^{(u, 0)\left(b^{\prime \prime}\right]}$, where

$$
\begin{aligned}
b^{\prime \prime} & =b^{\prime} v=a z^{-1} v=a v^{-1} u^{-1} \quad(\text { by (8)) } \\
& =b .
\end{aligned}
$$

This settles the reduction. Now let $v=1$. Then

$$
\begin{aligned}
C_{0}^{(u, 1)[b]} & =C_{0} u^{-1} \cdot u, 1 b^{-1(u, 1)} \\
& =\left(C_{0} u^{-1} u\right)\left(P\left(u^{-1}\right) b^{-1}\right) \quad(\text { by }(14)) \\
& =C_{0}\left(u^{-1}\left(u a^{-1}\right) u^{-1}\right)=C_{0} a^{-1} u^{-1}=C_{0}^{[a](u, 1)}
\end{aligned}
$$


and, for $x \in C$,

$$
\begin{aligned}
\bar{x}^{(u, 1)[b]} & =b \cdot_{u, 1} \bar{x}^{(u, 1)}{ }_{u, 1} b^{-1(u, 1)} \\
& =\left[\left\{(b u)\left(P\left(u^{-1}\right)(n(u) \bar{x})\right)\right\} u\right]\left[P\left(u^{-1}\right) b^{-1}\right] \\
& =\left[a\left(u^{-1} n(u) \bar{x}\right)\right]\left[a^{-1} u^{-1}\right] \\
& =\left[a(\bar{u} \bar{x}) a^{-1}\right] u^{-1} \quad(\text { by }(12)) \\
& =\overline{x u}^{[a]} u^{-1}=\bar{x}^{[a](u, 1)} .
\end{aligned}
$$

This completes the proof of Proposition 2.

A coordinate triple $\left(D, K, D_{0}\right)$ is said to be similar to $\left(C, J, C_{0}\right)$ if, for some $a \in C_{0} \cap C^{\times},\left(D, K, D_{0}\right)$ becomes isomorphic to an isotope of $\left(C, J, C_{0}\right)^{[a]}$. By Proposition 2, similarity defines an equivalence relation on the class of coordinate triples.

The above computations become almost trivial when dealing with involutions whose norms are all in the center. It is therefore of interest to construct examples of indecomposable finite dimensional properly alternative algebras with involution having all norms in the nucleus but not all norms in the center. This will be done at the end of the paper.

3. Isomorphisms of Jordan matrix algebras. By rephrasing and expanding Theorem 3 in [6], one arrives at the following result on isomorphisms of Jordan matrix algebras fixing the diagonal idempotents.

Proposition 3. Let $\left(C, J, C_{0}\right)$ and $\left(D, K, D_{0}\right)$ be coordinate triples, $m \geqslant 3$ an integer and $g=\operatorname{diag}\left(g_{1}, \ldots, g_{m}\right), h=\operatorname{diag}\left(h_{1}, \ldots, h_{m}\right)$ diagonal matrices admissible for $\left(C, J, C_{0}\right),\left(D, K, D_{0}\right)$, respectively, and satisfying $g_{1}=1, h_{1}=1$. Suppose

$$
\varphi: \mathrm{H}_{m}\left(C, J, C_{0}, g\right) \rightarrow \mathrm{H}_{m}\left(D, K, D_{0}, h\right)
$$

is an isomorphism such that $\varphi(1[i i])=1[i i]$ for $1<i<m$. Define $\rho: C \rightarrow D$ by

$$
\varphi\left(a[12]_{g}\right)=\rho(a)[12]_{h} \quad(a \in C)
$$

and $s, t \in D$ by

$$
\varphi\left(1[12]_{g}\right)=s[12]_{h}, \varphi\left(1[13]_{g}\right)=t[13]_{h} .
$$

Then $s, t \in D^{\times}$, and $\rho$ is an isomorphism from $\left(C, J, C_{0}\right)$ onto $\left(D, K, D_{0}\right)^{(u, v)}$ where $u=s^{-1} t, v=t^{-1}$.

Proof. (3) immediately implies that $s, t$ are invertible in $D$. Setting $w=u v=s^{-1}$, the calculations in the proof of Theorem 3 in [6] may be repeated verbatim to show that $\rho$ is an isomorphism from $C$ onto $D^{(u, v)}$ sending $C_{0}$ onto $D_{0}^{(u, v)}$; as a byproduct one also has $\varphi\left(g_{2}^{-1}[22]\right)=$ 
$s^{-1} s^{-1} h_{2}^{-1}[22]$. Using this as well as (3), (5), we obtain, for $a \in C$,

$$
\begin{aligned}
\rho(\bar{a})[12]_{h} & =\varphi\left(\bar{a}[12]_{g}\right)=\varphi\left(P_{g}\left(1[12]_{g}\right)\left\{1[11] a[12]_{g} g_{2}^{-1}[22]\right\}_{g}\right) \\
& =P_{h}\left(s[12]_{h}\right)\left\{1[11] \rho(a)[12]_{h} s^{-1} \bar{s}^{-1} h_{2}^{-1}[22]\right\}_{h} \\
& =P_{h}\left(s[12]_{h}\right) \rho(a) s^{-1} \bar{s}^{-1} h_{2}^{-1}[12]_{h} \\
& =s\left(h_{2} h_{2}^{-1} s^{-1} \bar{s}^{-1} \overline{\rho(a)}\right) s[12]_{h}=\overline{\rho(a) w} w^{-1}[12]_{h} \\
& =\overline{\rho(a)}(u, v)[12]_{h} .
\end{aligned}
$$

Hence $\rho$ respects the involutions, and Proposition 3 follows.

We would like to point out here that the arguments above together with [6, Theorem 3] immediately yield a new proof of Proposition 1(ii). However, this approach requires at least parts of the Coordinatization Theorem as well as the fact that $\mathrm{H}_{m}\left(C, J, C_{0}\right)$ carries the structure of a quadratic Jordan algebra, both of which can be established only by fairly long computations, and so a direct proof, independent of the Jordan theory, seems to be desirable.

As an immediate consequence of Proposition 3, we obtain a sufficient condition for two Jordan matrix algebras to be isotopic.

Corollary. Let $\left(C, J, C_{0}\right),\left(D, K, D_{0}\right)$ be similar coordinate triples and $m>3$ an integer. Then $\mathrm{H}_{m}\left(C, J, C_{0}\right)$ and $\mathrm{H}_{m}\left(D, K, D_{0}\right)$ are isotopic.

Proof. If $a \in C_{0} \cap C^{\times}$, the Jordan algebras $\mathrm{H}_{m}\left(C, J, C_{0}\right)$ and $\mathrm{H}_{m}\left(C, J^{[a]}, C_{0}^{[a]}, g\right)$, where $g=\operatorname{diag}\left(a^{-1}, \ldots, a^{-1}\right)$, are the same, so $\mathrm{H}_{m}\left(C, J, C_{0}\right)$ is isotopic to $\mathrm{H}_{m}\left(C, J^{[a]}, C_{0}^{[a]}\right)$. We may therefore assume from now on that $\left(D, K, D_{0}\right)=\left(C, J, C_{0}\right)^{(u, v)}$, for some $u, v \in C^{\times}$. Setting $w=u v$, the General Coordinatization Theorem leads to a coordinate triple $\left(B, I, B_{0}\right)$, a diagonal matrix $f=\operatorname{diag}\left(f_{1}, \ldots, f_{m}\right)$, admissible for $\left(B, I, B_{0}\right)$, as well as satisfying $f_{1}=1$, and an isomorphism $\eta: \mathrm{H}_{m}\left(C, J, C_{0}\right) \rightarrow \mathrm{H}_{m}\left(B, I, B_{0}, f\right)$ such that $\eta(1[i i])=1[i i] \quad(1<i<m), \eta\left(w^{-1}[12]\right)=1[12]_{f}, \quad \eta\left(v^{-1}[13]\right)=$ $1[13]_{f}$, and $\eta(1[1 i])=1[1 i]_{f}(4<i<m)$. Applying Proposition 3 to $\varphi=\eta^{-1}$, we obtain an induced isomorphism from $\left(B, I, B_{0}\right)$ onto $\left(C, J, C_{0}\right)^{(u, v)}=$ $\left(D, K, D_{0}\right)$. Hence $\mathrm{H}_{m}\left(D, K, D_{0}\right)$, being isomorphic to $\mathrm{H}_{m}(B, I, B)$, is isotopic to $\mathrm{H}_{m}\left(C, J, C_{0}\right)$.

4. Complete Jordan pairs. We wish to derive the converse of the preceding corollary under certain additional hypotheses on the coordinate triples involved. The appropriate framework for these hypotheses is the theory of Jordan pairs, due to Loos [4], which will be taken for granted in the sequel. However, we will have to make a few comments on the terminology and results in [7].

Accordingly, fixing a Jordan pair $V$ over $k, V$ is said to be connected in case any two orthogonal local idempotents in $V / \mathrm{rad} V$ are connected in the usual 
sense. We recall from [7] the following results.

LEMMA 1. Let $c$ be an idempotent in $V$ and suppose that $d^{+} \in V_{2}(c)^{+}$is invertible in $V_{2}(c)$, with inverse $d^{-} \in V_{2}(c)^{-}$. Then $d=\left(d^{+}, d^{-}\right)$is an idempotent in $V$ satisfying $V_{i}(d)=V_{i}(c)$ for $i=0,1,2$.

LEMMA 2. Let $c, e$ be idempotents and $h$ an automorphism of $V$ sending $e$ into $V_{2}(c)$. Then $h V_{2}(e) \subset V_{2}(c)$, and we have equality if and only if $h^{+} e^{+}$is invertible in $V_{2}(c)$.

ConJugacy Theorem. Let $V$ be connected and $\left(c_{1}, \ldots, c_{r}\right),\left(e_{1}, \ldots, e_{r}\right)$ be orthogonal systems of local idempotents in $V$, where $r$ is a positive integer. Then there exists an inner automorphism $h$ of $V$ such that $h V_{2}\left(e_{i}\right)=V_{2}\left(c_{i}\right)$ for $1 \leqslant i \leqslant r$.

As an immediate consequence of the Conjugacy Theorem (cf. Corollary 1 of the Main Theorem in [7]) one obtains that, if $V$ is connected, any two frames in $V$ have the same (finite or infinite) length, called the capacity of $V$.

For technical reasons, it will be convenient to single out by intrinsic properties a class of Jordan pairs which embraces all local Jordan pairs as well as all connected ones having dcc on principal inner ideals and containing invertible elements. We formalize as follows: $V$ is said to be complete if

C1. $V$ is connected and of finite capacity;

C2. for each frame $\left(c_{1}, \ldots ; c_{r}\right)$ in $V$, we have

$$
V=V_{2}\left(\sum_{i=1}^{r} c_{i}\right) \text {. }
$$

Obviously, every complete Jordan pair contains invertible elements, and every local Jordan pair is complete. On the other hand, suppose $V$ is connected, has dcc on principal inner ideals and contains invertible elements. Then $\mathrm{Cl}$ trivially holds. Also, $V$ contains an idempotent $e$ which satisfies $V=V_{2}(e)$ and is therefore maximal. Given a frame $\left(c_{1}, \ldots, c_{r}\right)$ in $V$, the idempotent $c=\Sigma c_{i}$ is maximal as well, and, by Corollary 4 of the Main Theorem in [7], there exists an inner automorphism $h$ of $V$ satisfying $h V_{2}(c)$ $=V_{2}(e)=V$. This proves $\mathrm{C} 2$, and $V$ is complete.

LEMMA 3. Let $c, d$ be local idempotents of $V$ and $W=V_{1}(c) \cap V_{1}(d)$. Then $c$ and $d$ are connected if and only if $\mathrm{rad} W \neq W$.

PRoof. $c$ and $d$ are connected if and only if their canonical images in $V / \operatorname{rad} V$ are connected. Hence, as $\operatorname{rad} W=W \cap \operatorname{rad} V$, we may assume that $V$ is semisimple. Then $c$ and $d$ are completely primitive, and if they are also connected, we trivially have $W \neq\{0\}$. Conversely, suppose $c$ and $d$ are not connected. Then we may apply [3, III, Lemma 2, pp. 3.22-3.23] to the 
quadratic Jordan algebra $g=V_{2}(c+d)_{z}^{+}\left(z=c^{-}+d^{-}\right)$and its supplementary set $\left\{c^{+}, d^{+}\right\}$of orthogonal completely primitive idempotents to conclude that $W^{+}$consists of absolute zero divisors in $q$. Therefore $(x, y)$ is nilpotent, hence quasi-invertible, for $x \in W^{+}, y \in V_{2}(c+d)^{-}$, and it follows $W^{+} \subset \operatorname{rad} V_{2}(c+d)^{+}=\{0\}$. The same argument with + and interchanged yields $W^{-}=\{0\}$, and the proof is complete.

LEMMA 4. Suppose $c, d$ are orthogonal connected idempotents in $V$. Let $\left(c_{1}, \ldots, c_{r}\right),\left(c_{r+1}, \ldots, c_{s}\right)$ be orthogonal systems of pairwise connected local idempotents in $V_{2}(c), V_{2}(d)$, respectively, satisfying

$$
V_{2}(c)=V_{2}\left(\sum_{i=1}^{r} c_{i}\right), \quad V_{2}(d)=V_{2}\left(\sum_{i=r+1}^{s} c_{i}\right) .
$$

Then, for all $i=1, \ldots, r, j=r+1, \ldots, s$, the idempotents $c_{i}$ and $c_{j}$ are connected.

Proor. By transitivity of connectedness, it suffices to show that there exist indices $i=1, \ldots, r, j=r+1, \ldots, s$ for which $c_{i}$ and $c_{j}$ are connected. We may further assume $V=V_{2}(c+d)$, put

$$
c^{\prime}=\sum_{k=1}^{r} c_{i}, d^{\prime}=\sum_{i=r+1}^{s} c_{i}
$$

and denote by $V_{i j}(1<i, j<s)$ the Peirce subpairs of $V$ relative to $\left(c_{1}, \ldots, c_{s}\right)$. Then, by Lemma 1 , we have

$$
W=V_{1}(c) \cap V_{1}(d)=V_{1}\left(c^{\prime}\right) \cap V_{1}\left(d^{\prime}\right)=\sum_{i<r<j} V_{i j} .
$$

$c$ and $d$ being connected, $W$ certainly cannot be a radical pair, and so there are $i=1, \ldots, r, j=r+1, \ldots, s$ satisfying $\operatorname{rad} V_{i j} \neq V_{i j}$. Now Lemma 3 shows that $c_{i}$ and $c_{j}$ are connected.

Proposition 4. Let $X=\left(e_{1}, \ldots, e_{m}\right)$ be an orthogonal system of pairwise connected idempotents in $V$ such that $V=V_{2}(e)$, where $e=\Sigma e_{i}$, and $V_{2}\left(e_{1}\right)$ is complete. Then $V$ is complete, and, if $X^{\prime}=\left(e_{1}^{\prime}, \ldots, e_{m}^{\prime}\right)$ is a system with the same properties as $X$, there exists an inner automorphism $h$ of $V$ satisfying $h V_{2}\left(e_{i}^{\prime}\right)=V_{2}\left(e_{i}\right)$ for $1<i<m$.

Proof. For $i=1, \ldots, m, V_{2}\left(e_{i}\right)$ is isomorphic to $V_{2}\left(e_{1}\right)[7$, Theorem 1] and hence complete; in particular, $r$, the capacity of $V_{2}\left(e_{i}\right)$, is independent of $i$. Let $\left(c_{(i-1) r+1}, \ldots, c_{i r}\right)$ be a frame in $V_{2}\left(e_{i}\right)$ and put

$$
d_{i}=\sum_{j=1}^{r} c_{(i-1) r+j}, \quad d=\sum_{i=1}^{m} d_{i}=\sum_{j=1}^{s} c_{j}
$$

where $s=r m$. As $V_{2}\left(e_{i}\right)$ is complete, we have $V_{2}\left(e_{i}\right)=V_{.2}\left(d_{i}\right)$, which, because 
of Lemma 1, implies $V_{2}(d)=V_{2}(e)=V$. Hence $Y=\left(c_{1}, \ldots, c_{s}\right)$ is a frame in $V$ consisting, by Lemma 4, of pairwise connected local idempotents. Now Theorem 3 in [7] shows that $V$ is connected, having capacity $s$. In particular, given another frame, $Y^{\prime}=\left(c_{1}^{\prime}, \ldots, c_{s}^{\prime}\right)$, in $V$, the Conjugacy Theorem leads to some inner automorphism $h$ of $V$ sending $V_{2}\left(c_{j}^{\prime}\right)$ onto $V_{2}\left(c_{j}\right), j=1, \ldots, s$, which implies $h V_{2}\left(\sum_{j=1}^{s} c_{j}^{\prime}\right)=V_{2}(d)=V$, so $V$ is complete. Also, repeating the construction above with $X^{\prime}$ in place of $X$ shows that $r$ is the capacity of $V_{2}\left(e_{i}^{\prime}\right)(1<i<m)$ and leads to a frame $Y^{\prime}=\left(c_{1}^{\prime}, \ldots, c_{s}^{\prime}\right)$ in place of $Y$. Hence, setting

$$
d_{i}^{\prime}=\sum_{j=1}^{r} c_{(i-1) r+j}^{\prime} \quad(1 \leqslant i \leqslant m)
$$

and choosing $h$ as before, gives as desired.

$$
h V_{2}\left(e_{i}^{\prime}\right)=h V_{2}\left(d_{i}^{\prime}\right)=V_{2}\left(d_{i}\right)=V_{2}\left(e_{i}\right) \quad(1 \leqslant i \leqslant m),
$$

5. The Isotopy Theorem and examples. Let $\left(C, J, C_{0}\right)$ be a coordinate triple. Obviously, $C_{0}$ makes up a unital subalgebra of $C^{+}$. In accordance with the terminology of [4], the corresponding Jordan pair will be written as $\left(C_{0}, C_{0}\right)$. We are interested in coordinate triples where this Jordan pair is complete. According to 4 and [7, Proposition 2], this assumption holds automatically when $C_{0}$ is local or has dcc on principal inner ideals and becomes simple after reducing modulo its radical. We are now ready to prove the

IsOTOPY THEOREM. Let $\left(C, J, C_{0}\right)$ and $\left(D, K, D_{0}\right)$ be coordinate triples such that the Jordan pairs $\left(C_{0}, C_{0}\right)$ and $\left(D_{0}, D_{0}\right)$ are complete. Then, given an integer $m>3$, the Jordan matrix algebras $\mathrm{H}_{m}\left(C, J, C_{0}\right)$ and $\mathrm{H}_{m}\left(D, K, D_{0}\right)$ are isotopic if and only if $\left(C, J, C_{0}\right)$ and $\left(D, K, D_{0}\right)$ are similar.

Proof. The sufficiency of this condition has been established in the corollary of Proposition 3. Conversely, write $V, W$ for the Jordan pair attached to $A=\mathrm{H}_{m}\left(C, J, C_{0}\right), B=\mathrm{H}_{m}\left(D, K, D_{0}\right)$, respectively. Setting $e_{i}=$ (1[ii], $1[i i]) \in B \times B$ for $1 \leqslant i<m, X=\left(e_{1}, \ldots, e_{m}\right)$ is an orthogonal system of a pairwise connected idempotents in $W$ such that $W=W_{2}\left(\sum e_{i}\right)$ and $W_{2}\left(e_{1}\right)=\left(D_{0}, D_{0}\right)$, so, by hypothesis, $W_{2}\left(e_{1}\right)$ is complete. Hence Proposition 4 implies that $W$ is complete. Now suppose $A$ and $B$ are isotopic. Then $V$ and $W$ are isomorphic. Choosing an isomorphism $\Phi: V \rightarrow W$, setting $c_{i}=(1[i i], 1[i i]) \in A \times A$ for $1<i<m$ and observing the completeness of $\left(C_{0}, C_{0}\right)$, we see that Proposition 4 applies to $X^{\prime}=\left(\Phi\left(c_{1}\right), \ldots, \Phi\left(c_{m}\right)\right)$. Hence there is no harm in assuming $\Phi V_{2}\left(c_{i}\right)=W_{2}\left(e_{i}\right), i=1, \ldots, m$, which amounts to the existence of $g_{1}, \ldots, g_{m} \in D_{0} \cap D^{\times}$such that $\Phi^{+} 1[i i]=$ $g_{i}[i i]$. As $\Phi$ is an isomorphism of Jordan pairs, $\Phi^{+}$is an isomorphism from $A$ onto $B^{\left(g^{-1}\right)}, g=\operatorname{diag}\left(g_{1}, \ldots, g_{m}\right)$, so $\Phi^{+}$followed by right multiplication by $g^{-1}$ makes up an isomorphism $\varphi$ from $A$ onto $\mathrm{H}_{m}\left(D, K, D_{0}, g\right)$ satisfying 
$\varphi(1[i i])=1[i i]$. Setting $a=g_{1}$ and replacing $\left(D, K, D_{0}\right)$ by the similar coordinate triple $\left(D, K^{[a]}, D_{0}^{[a]}\right)$ as well as $g$ by $g a^{-1}$ allows us to assume $g_{1}=1$. Now Proposition 3 shows that $\left(C, J, C_{0}\right)$ and $\left(D, K, D_{0}\right)$ are isotopic. This completes the proof.

We close the paper by discussing some examples. Let $\left(C, J, C_{0}\right)$ be a coordinate triple and suppose first that $C$ is associative. Then isotopy reduces to isomorphism, and hence another coordinate triple is similar to $\left(C, J, C_{0}\right)$ if and only if, for some $a \in C_{0} \cap C^{\times}$, it is isomorphic to $\left(C, J, C_{0}\right)^{[a]}$. Next suppose that $k$ is a field, and let $C$ be a Cayley algebra over $k$. Then, as $\mathrm{N}(C)=k 1, J$ agrees with the standard involution of $C$, and all isotopes of $C$ are isomorphic [8, p. 554]. Hence the isomorphism class of $C$ determines the similarity class of $\left(C, J, C_{0}\right)$, and the Isotopy Theorem yields Jacobson's result described in the introduction. More specifically, let $k$ be the quotient field of a local ring $R$ and $M$ a $J$-invariant order in $C$ (containing 1 ). Then $\left(M,\left.J\right|_{M}, R 1\right)$ is a coordinate triple over $R$ to which the Isotopy Theorem may be applied. Finally, if $k$ is a field of characteristic not two, let $A$ be a Cayley algebra over $k$ with standard involution $x \mapsto \bar{x}$ and $B$ a quaternion subalgebra of $A$. Then $B, A$ may canonically be regarded as alternative bimodules $P, Q$, respectively, for $B$, and the split null extension $C=B \oplus N$, where $N$ is the $B$-bimodule $P \oplus Q$, becomes a unital alternative algebra of dimension 16 , with center $k 1 \oplus k 1 \oplus k 1$ and nucleus $k 1 \oplus P \oplus P$. Also, it is easily verified that the rule $(a, p, q) \mapsto(\bar{a},-\bar{p}, \bar{q})$ for $a \in B, p \in P, q \in Q$ defines an involution $J$ of $C$ having all norms in the nucleus but not all norms in the center. More precisely, $\mathrm{H}(C, J)=k 1 \oplus P_{0} \oplus k 1, P_{0}$ corresponding to the pure quaternions, and it follows that $(C, J, \mathrm{H}(C, J))$ is a coordinate triple to which the Isotopy Theorem applies.

\section{REFERENCES}

1. J. R. Faulkner, Octonian planes defined by quadratic Jordan algebras, Mem. Amer. Math. Soc. No. 104, 1970.

2. N. Jacobson, Structure and representations of Jordan algebras, Amer. Math. Soc. Colloq. Publ., vol. 39, Amer. Math. Soc., Providence, R.I., 1968.

3. ___ Lectures on quadratic Jordan algebras, Tata Institute Lecture Notes, Bombay, 1969.

4. O. Loos, Jordan pairs, Lecture Notes in Math., vol. 460, Springer-Verlag, Berlin and New York, 1975.

5. K. McCrimmon, A general theory of Jordan rings, Proc. Nat. Acad. Sci. U.S.A. 56 (1966), 1072-1079.

6. ___ Homotopes of alternative algebras, Math. Ann. 191 (1971), 253-262.

7. H. P. Petersson, Conjugacy of idempotents in Jordan pairs, Comm. Algebra (to appear).

8. R. D. Schafer, Alternative algebras over an aribtrary field, Bull. Amer. Math. Soc. 49 (1943), 549-555.

MATHematisches Institut der Universität, RoXeler Strasse 64, D-44 MüNSTER, BUNDESREPUBLIK DEUTSCHLAND 